\title{
ON THE CARLESON MEASURE CHARACTERIZATION OF BMOA FUNCTIONS ON THE UNIT BALL
}

\author{
MIROLJUB JEVTIĆ
}

(Communicated by Paul S. Muhly)

\begin{abstract}
An equivalent form of the well-known Carleson measure condition that characterizes BMOA functions on the unit ball is given. As an application, it is proved that the Toeplitz operator $T_{\bar{\varphi}}, \varphi \in H^{\infty}(S)$, is bounded on the Hardy-Sobolev space $H_{\alpha}^{1}, 0<\alpha<\infty$.
\end{abstract}

\section{INTRODUCTION}

Let $B$ denote the unit ball in $C^{n}, n \geq 1$, and $m$ the $2 n$-dimensional Lebesgue measure on $B$ normalized so that $m(B)=1$, while $\sigma$ is the normalized surface measure on its boundary $S$.

The Hardy space $H^{p}, 1 \leq p<\infty$, is defined as that space of holomorphic functions $f$ on $B(f \in H(B))$ satisfying

$$
\|f\|_{p}^{p}=\sup _{0<r<1} \int_{S}|f(r \xi)|^{p} d \sigma(\xi)<\infty .
$$

A function $f \in H(B)$ is said to belong to $H^{\infty}$ if

$$
\|f\|_{\infty}=\sup _{z \in B}|f(z)|<\infty .
$$

We write BMOA for the space consisting of functions in $H^{2}$ of bounded mean oscillation. These spaces are discussed in more detail by Coifman, Rochberg, and Weiss [4].

For a holomorphic function $f$ on $B$ with homogeneous expansion $f=$ $\sum_{k=0}^{\infty} f_{k}$ the radial fractional derivative of order $\beta>0$ is defined by

$$
D^{\beta} f(z)=\sum_{k=0}^{\infty}(k+1)^{\beta} f_{k}(z) ;
$$

(thus for $\beta=1, D^{1} f=f+R f$, where $R$ denotes the radial derivative operator defined by $R f(z)=\sum_{j=1}^{n} z_{j}\left(\partial f / \partial z_{j}(z)\right)$.

Received by the editors May 8, 1990 and, in revised form, September 7, 1990.

1980 Mathematics Subject Classification (1985 Revision). Primary 32A35.

Key words and phrases. Carleson measures, BMOA spaces, Toeplitz operators, Hardy-Sobolev spaces.

(C) 1992 American Mathematical Society $0002-9939 / 92 \$ 1.00+\$ .25$ per page 
A positive measure $\nu$ on $B$ is called a Carleson measure if

$$
\nu(Q(\xi, \delta)) \leq C \delta^{n}, \quad \xi \in S, \delta>0,
$$

where $Q(\xi, \delta)=\{z \in B:|1-\langle z, \xi\rangle|<\delta\}$ are nonisotropic balls.

Here and elsewhere constants are denoted by $C$, which may indicate a different constant from one occurrence to the next.

Any unexplained notation is as in [9].

In this note we consider measures $\mu_{\alpha, f}, 0<\alpha<\infty, f \in H(B)$, defined by $d \mu_{\alpha, f}(r \xi)=\left|D^{\alpha} f(r \xi)\right|^{2}(1-r)^{2 \alpha-1} d r d \sigma(\xi), 0<r<1, \xi \in S$. Our main result is the following theorem.

Theorem 1. Let $f \in H(B)$ and $0<\alpha<\infty$. If $\mu_{\alpha, f}$ is a Carleson measure on $B$ for some $\alpha$, then $\mu_{\alpha, f}$ is a Carleson measure on $B$ for all $\alpha$.

Our work was motivated by the result:

A function $g \in H^{2}$ belongs to BMOA if and only if a measure $\mu_{1, g}$ is a Carleson measure.

If $g \in \mathrm{BMOA}$, to show that $\mu_{1, g}$ is a Carleson measure we argue as on pp. 146-147 of [5] making the obviously necessary changes. Conversely, if $\mu_{1, g}$ is a Carleson measure then using Corollary 5.3 [2], "Fefferman's trick" as in the proof of implication (c) $\rightarrow(d)$, Theorem 5 [1, p. 26] and Theorem $V$ [4] we find that $g \in \mathrm{BMOA}$.

From this and Theorem 1 we have a new characterization of BMOA functions on the unit ball.

Theorem 2. For $g \in H(B)$ the following are equivalent:

(i) $g \in \mathrm{BMOA}$,

(ii) For some $\alpha>0,\left|D^{\alpha} g(z)\right|^{2}\left(1-|z|^{2}\right)^{2 \alpha-1} d m(z)$ is a Carleson measure on $B$,

(iii) Same as (ii) but for every $\alpha>0$.

As an application we consider the Toeplitz operators on the Hardy-Sobolev spaces $H_{\alpha}^{1}, 0<\alpha<\infty$.

For $\varphi \in L^{\infty}(\sigma)$ the Toeplitz operator with symbol $\varphi, T_{\varphi}$, is defined for $z \in B$ by

$$
T_{\varphi} f(z)=C[\varphi f](z), \quad f \in L^{1}(\sigma),
$$

where

$$
C[\varphi f](z)=\int_{S}(1-\langle z, \xi\rangle)^{-n} \varphi(\xi) f(\xi) d \sigma(\xi)
$$

is the Cauchy integral of a function $\varphi f$.

The Hardy-Sobolev space is defined as

$$
H_{\alpha}^{p}=\left\{f \in H(B):\left\|D^{\alpha} f\right\|_{p}<\infty\right\}, \quad 1 \leq p \leq \infty, 0<\alpha<\infty .
$$

If $f \in H_{\alpha}^{p}, 0 \leq \alpha<\infty, 1 \leq p \leq \infty,\left(H_{0}^{p}=H^{p}\right)$, the limits $f(\xi)=$ $\lim _{r \rightarrow 1} f(r \xi)$ exist for $\sigma$-almost every $\xi \in S$ and identify $H_{r}^{p}$ isometrically with a closed subspace $H_{\alpha}^{p}(S)$ of $L^{1}(\sigma)$ (see [9]).

It is well known that every $T_{\varphi}$ is a bounded linear operator from $H^{p}(S)$, $1<p<\infty$, to $H^{p}$, while it is not true that every $T_{\bar{\varphi}}$ carries $H^{1}(S)$ to $H^{1}$, even when $\varphi \in H^{\infty}(S)$. In this note we show that if a domain of $T_{\bar{\varphi}}, \varphi \in H^{\infty}(S)$ 
is restricted to the smaller class $H_{\alpha}^{1}(S), 0<\alpha<\infty$, then $T_{\bar{\varphi}}$ is a bounded operator.

Theorem 3. Every $T_{\bar{\varphi}}$, with symbol $\varphi \in H^{\infty}(S)$, is a bounded linear operator from $H_{\alpha}^{1}(S)$ to $H_{\alpha}^{1}$ for all $0<\alpha<\infty$.

If $n=1$ and $\alpha$ is a positive integer, then this theorem is due to $\mathrm{F}$. Shamoyan [10].

\section{Proof of Theorem 1}

For $z \in B$ and $0<r<1$, let $E(z, r)=\left\{w \in B:\left|\varphi_{z}(w)\right|<r\right\}$, where $\varphi_{z}$ denotes the standard automorphism of $B$ taking 0 to $z$.

In proving Theorem 1 our main tool is the following lemma, which was proved in [6] by a different method in case $n=1$.

Lemma 2.1. Let $k$ be a positive integer and let $r$ be fixed $0<r<1$. There is a constant $C$, depending only on $k$ and $r$ such that if $f \in H(B)$, then

$$
\left|D^{k} f(z)\right|^{2} \leq \frac{C(1-|z|)^{-2 k}}{m(E(z, r))} \int_{E(z, r)}|f(w)|^{2} d m(w), \quad z \in B .
$$

Proof. We give a detailed proof only in case $k=1$. The general case is technically rather complicated but requires no new ideas. Our proof in the case $k>1$ is rather sketchy.

If $\alpha=\left(\alpha_{1}, \alpha_{2}, \ldots, \alpha_{n}\right)$ is a multi-index of nonnegative integers with order $|\alpha|=\alpha_{1}+\alpha_{2}+\cdots+\alpha_{n}$ then $\partial^{\alpha}$ denotes the monomial $\partial^{|\alpha|} / \partial z_{1}^{\alpha_{1}} \partial z_{2}^{\alpha_{2}} \cdots \partial z_{n}^{\alpha_{n}}$.

We use the estimate

$$
\left|\partial^{\alpha} g(z)\right| \leq C(1-|z|)^{-k}\|g\|_{\infty}, \quad|\alpha| \leq k, g \in H^{\infty},
$$

(see [3]).

By the formula 4.2.4(5), [9, p. 52],

$$
f(z)=\int_{B} f(w) h\left(\varphi_{z}(w)\right)\left(1-|w|^{2}\right)^{-n-1} d m(w),
$$

where $h$ is a radial function that belongs to $C^{\infty}(B)$ with compact support in $B$.

Since $h$ is radial and $\left|\varphi_{z}(w)\right|=\left|\varphi_{w}(z)\right|$, we have

$$
f(z)=\int_{B} f(w) h\left(\varphi_{w}(z)\right)\left(1-|w|^{2}\right)^{-n-1} d m(w) .
$$

Denote the components of $\varphi_{w}$ by $\varphi_{1}(\cdot, w), \varphi_{2}(\cdot, w), \ldots, \varphi_{n}(\cdot, w)$. Since these are holomorphic in $B$ with $\left\|\varphi_{j}(\cdot, w)\right\|_{\infty}=1, j=1, \ldots, n$, for all $w \in B$, we have by $(2.2)$

$$
\left|\frac{\partial \varphi_{j}(z, w)}{\partial z_{i}}\right| \leq C(1-|z|)^{-1}, \quad z, w \in B, i, j=1,2 \ldots, n .
$$

Now the chain rule gives

$$
\begin{aligned}
\left|\frac{\partial f(z)}{\partial z_{j}}\right| & =\left|\int_{B} f(w) h^{\prime}\left(\varphi_{w}(z)\right)\left(\sum_{i=1}^{n} \frac{\partial \varphi_{i}(z, w)}{\partial z_{j}} \frac{\bar{\varphi}_{i}(z, w)}{2\left|\varphi_{w}(z)\right|}\right)\left(1-|w|^{2}\right)^{-n-1} d m(w)\right| \\
& \leq C(1-|z|)^{-1} \int_{B}|f(w)|\left|h^{\prime}\left(\varphi_{w}(z)\right)\right|\left(1-|w|^{2}\right)^{-n-1} d m(w) .
\end{aligned}
$$


By a suitable choice of a function $h$, we obtain $\left|\frac{\partial f(z)}{\partial z_{j}}\right| \leq C(1-|z|)^{-1} \int_{E(z, r)}|f(w)|\left(1-|w|^{2}\right)^{-n-1} d m(w), \quad j=1, \ldots, n$.

Thus,

$$
\left|D^{1} f(z)\right| \leq C(1-|z|)^{-1} \int_{E(z, r)}|f(w)|\left(1-|w|^{2}\right)^{-n-1} d m(w) .
$$

In [8] it is proved that for all $w \in E(z, r)$, we have

$$
\frac{1-r}{1+r} \leq \frac{1-|z|^{2}}{1-|w|^{2}} \leq \frac{1+r}{1-r} \text {. }
$$

It is also the case that $m(E(z, r)) \cong\left(1-|z|^{2}\right)^{n+1}$ (see [9, 2.2.7]) where the symbol $\cong$ indicates that the quantities have ratios bounded and bounded away from zero as $z$ varies.

Hence from the above estimates, we conclude that

$$
\left|D^{1} f(z)\right| \leq \frac{C(1-|z|)^{-1}}{m(E(z, r))} \int_{E(z, r)}|f(w)| d m(w) .
$$

Similarly, by differentiation of formula (2.3) and using (2.2), we find that

$$
\left|D^{k} f(z)\right| \leq \frac{C(1-|z|)^{-k}}{m(E(z, r))} \int_{E(z, r)}|f(w)| d m(w) .
$$

Now (2.1) follows by Jensen's inequality.

Proof of Theorem 1. We write $\mu_{\alpha}$ instead of $\mu_{\alpha, f}$. Suppose $\mu_{\alpha}$ is a Carleson measure on $B$ and let $0<\beta<\alpha<\infty$.

Let $\xi \in S$ and $\delta>0$. From [3] we see that

$$
\left|D^{\beta} f(z)\right| \leq C \int_{B} \frac{\left|D^{\alpha} f(w)\right|(1-|w|)^{s-1}}{|1-\langle w, z\rangle|^{n+s-\alpha+\beta}} d m(w)
$$

for any $s>0$ that satisfies $n+s>\alpha-\beta$. Thus we may suppose that $s>2 \alpha$. By standard estimates (see $[9$, p. 17])

$$
\int_{B} \frac{(1-|w|)^{s-1}}{|1-\langle w, z\rangle|^{n+s+\beta}} d m(w) \leq C \int_{0}^{1} \frac{(1-r)^{s-1}}{(1-r|z|)^{s+\beta}} d r \leq C(1-|z|)^{-\beta}, \quad z \in B .
$$

Thus by Holder's inequality

$$
\begin{aligned}
& \int_{Q(\xi, \delta)} d \mu_{\beta}(z) \\
& \leq C \int_{Q(\xi, \delta)}(1-|z|)^{2 \beta-1}\left(\int_{B} \frac{\left|D^{\alpha} f(w)\right|^{2}(1-|w|)^{s-1}}{|1-\langle w, z\rangle|^{n+s-2 \alpha+\beta}} d m(w) \int_{B} \frac{(1-|w|)^{s-1} d m(w)}{|1-\langle w, z\rangle|^{n+s+\beta}}\right) d m(z) \\
& \leq C \int_{Q(\xi, \delta)}(1-|z|)^{\beta-1}\left(\int_{Q(\xi, 2 \delta)} \frac{\left|D^{\alpha} f(w)\right|^{2}(1-|w|)^{s-1} d m(w)}{|1-\langle w, z\rangle|^{n+s-2 \alpha+\beta}}\right) d m(z) \\
& \quad+C \int_{Q(\xi, \delta)}(1-|z|)^{\beta-1}\left(\sum_{j} \int_{A_{j}} \frac{\left|D^{\alpha} f(w)\right|^{2}(1-|w|)^{s-1} d m(w)}{|1-\langle w, z\rangle|^{n+s+\beta-2 \alpha}}\right) d m(z)=I_{1}+I I_{2},
\end{aligned}
$$


where $A_{j}=\left\{w \in B: 2^{j} \delta \leq|1-\langle w, \xi\rangle|<2^{j+1} \delta\right\}, j=1,2$. As above, we have

$$
\begin{aligned}
& \int_{B} \frac{(1-|z|)^{\beta-1} d m(z)}{|1-\langle w, z\rangle|^{n+s+\beta-2 \alpha}} \\
& \quad \leq C \int_{0}^{1} \frac{(1-r)^{\beta-1}}{(1-|w| r)^{s+\beta-2 \alpha}} d r \leq C(1-|w|)^{2 \alpha-s}, \quad w \in B .
\end{aligned}
$$

(Note that here we need $s>2 \alpha$ ). Hence by Fubini's theorem

$$
\begin{aligned}
I_{1} & \leq C \int_{Q(\xi, 2 \delta)}\left|D^{\alpha} f(w)\right|^{2}(1-|w|)^{s-1}\left(\int_{B} \frac{(1-|z|)^{\beta-1} d m(z)}{|1-\langle w, z\rangle|^{n+s+\beta-2 \alpha}}\right) d m(w) \\
& \leq C \int_{Q(\xi, 2 \delta)} d \mu_{\alpha}(w) \leq C \delta^{n},
\end{aligned}
$$

since $\mu_{\alpha}$ is a Carleson measure.

Notice that if $z \in Q(\xi, \delta)$ and $w \in A_{j}$, we have

$$
|1-\langle z, w\rangle|^{1 / 2} \geq|1-\langle w, \xi\rangle|^{1 / 2}-|1-\langle z, \xi\rangle|^{1 / 2} \geq \frac{1}{2}(\sqrt{2}-1) 2^{j / 2} \delta^{1 / 2} .
$$

Hence

$$
\begin{aligned}
I_{2} & \left.\leq C \sum_{j}\left(2^{j} \delta\right)^{-n-\beta} \int_{A_{j}}\left|D^{\alpha} f(w)\right|^{2}(1-|w|)^{2 \alpha-1} d m(w)\right) \int_{Q(\xi, \delta)}(1-|z|)^{\beta-1} d m(z) \\
& \leq C \delta^{n+\beta} \sum_{j}\left(2^{j} \delta\right)^{-\beta} \leq C \delta^{n}
\end{aligned}
$$

Here we have used the estimate $\int_{Q(\xi, \delta)}(1-|z|)^{\beta-1} d m(z) \leq C(\beta) \delta^{n+\beta}$ (see $[3,9])$.

Now let $0<\alpha<\beta<\infty$. Choose a positive integer $k$ so that $k+\alpha>\beta$. To finish the proof of Theorem 1, it is sufficient to prove that $\mu_{\alpha+k}$ is a Carleson measure on $B$.

Let $\xi \in S, \delta>0$, and $0<r<1$. One can easily verify that there exists a constant $k=k(r)$ such that $E(z, r) \subseteq Q(\xi, k \delta)$ whenever $z \in Q(\xi, \delta)$. Using this fact, Lemma 2.1, and Fubini's theorem, we find that

$$
\begin{aligned}
\int_{Q(\xi, \delta)} & d \mu_{\alpha+k}(t \eta) \\
& \leq C \int_{Q(\xi, \delta)}\left(\int_{E(t \eta, r)}\left|D^{\alpha} f(w)\right|^{2}(1-|w|)^{-2 k-n-1} d m(w)\right)(1-t)^{2(\alpha+k)-1} d t d \sigma(\eta) \\
& \leq C \int_{Q(\xi, \delta)}\left(\int_{Q(\xi, k \delta)} \chi_{E(t \eta, r)}(w)\left|D^{\alpha} f(w)\right|^{2}(1-|w|)^{2 \alpha-n-2} d m(w)\right) d t d \sigma(\eta) \\
& =C \int_{Q(\xi, k \delta)}\left|D^{\alpha} f(w)\right|^{2}(1-|w|)^{2 \alpha-n-2} d m(w) \int_{Q(\xi, \delta)} \chi_{E(w, r)}(t \eta) d t d \sigma(\eta) \\
& \leq C \int_{Q(\xi, k \delta)} d \mu_{\alpha}(w) \leq C \delta^{n},
\end{aligned}
$$

since $\mu_{\alpha}$ is a Carleson measure. 


\section{TOEPLITZ OPERATORS}

The proof of Theorem 3 is facilitated by the next lemma.

Lemma 3.1. Let $\phi \in L^{\infty}(\sigma),\|\phi\|_{\infty}=1$ and $0<\alpha<\infty$. Then there exists a constant $C>0$ depending only on $\alpha$, such that

$$
\int_{0}^{1} \int_{S}\left|D^{\alpha+1} f(r \xi)\left\|D^{\alpha} C[\phi](r \xi) \mid(1-r)^{\alpha} d r d \sigma(\xi) \leq C\right\| D^{\alpha} f \|_{1},\right.
$$

for all $f \in H_{\alpha}^{1}$.

Proof. Since $D^{\alpha} f \in H^{1}$, there are functions $f_{k}, g_{k} \in H^{2}$ such that

$$
D^{\alpha} f=\sum_{k=1}^{\infty} f_{k} g_{k} \quad \text { and } \quad \sum_{k=1}^{\infty}\left\|f_{k}\right\|_{2}\left\|g_{k}\right\|_{2} \leq C\left\|D^{\alpha} f\right\|_{1}
$$

(see [4]). Hence

$$
\begin{aligned}
D^{\alpha+1} f & =D^{1}\left(D^{\alpha} f\right)=D^{\alpha} f+\sum_{j=1}^{n} z_{j} \frac{\partial\left(D^{\alpha} f\right)}{\partial z_{j}} \\
& =D^{\alpha} f+\sum_{j=1}^{n} \sum_{k=1}^{\infty} z_{j} \frac{\partial\left(f_{k} g_{k}\right)}{\partial z_{j}}=\sum_{k=1}^{\infty}\left(D^{1} f_{k}\right) g_{k}+\sum_{k=1}^{\infty} f_{k}\left(D^{1} g_{k}\right)-D^{\alpha} f .
\end{aligned}
$$

By Hölder's inequality

$$
\int_{0}^{1} \int_{S}\left|D^{1} f_{k}(r \xi)\right|\left|g_{k}(r \xi)\right|\left|D^{\alpha} C[\phi](r \xi)\right|(1-r)^{\alpha} d r d \sigma(\xi) \leq I\left(g_{k}\right) J\left(f_{k}\right),
$$

where

$$
I\left(g_{k}\right)=\left\{\int_{0}^{1} \int_{S}\left|g_{k}(r \xi)\right|^{2}\left|D^{\alpha} C[\phi](r \xi)\right|^{2}(1-r)^{2 \alpha-1} d r d \sigma(\xi)\right\}^{1 / 2}
$$

and

$$
J\left(f_{k}\right)=\left\{\int_{0}^{1} \int_{S}\left|D^{1} f_{k}(r \xi)\right|^{2}(1-r) d \sigma(\xi) d r\right\}^{1 / 2} .
$$

Since $\phi \in L^{\infty}(\sigma), C[\phi] \in$ BMOA and BMOA norm of $C[\phi]$ is at most $\|\phi\|_{\infty}=1$. Hence by using Theorem 2 , we conclude that $I\left(g_{k}\right) \leq C\left\|g_{k}\right\|_{2}$.

An integration in polar coordinates shows that $J\left(f_{k}\right) \cong\left\|f_{k}\right\|_{2}$.

Therefore,

$$
\begin{gathered}
\int_{0}^{1} \int_{S}\left|\sum_{k=1}^{\infty} D^{1} f_{k}(r \xi) g_{k}(r \xi)\right|\left|D^{\alpha} C[\phi](r \xi)\right|(1-r)^{\alpha} d r d \sigma(\xi) \\
\quad \leq C \sum_{k=1}^{\infty}\left\|f_{k}\right\|_{2}\left\|g_{k}\right\|_{2} \leq C\left\|D^{\alpha} f\right\|_{1},
\end{gathered}
$$

by (3.2).

By symmetry we have

$$
\int_{0}^{1} \int_{S}\left|\sum_{k=1}^{\infty}\left(D^{1} g_{k}\right)(r \xi) f_{k}(r \xi)\right|\left|D^{\alpha} C[\phi](r \xi)\right|(1-r)^{\alpha} d r d \sigma(\xi) \leq C\left\|D^{\alpha} f\right\|_{1}
$$


Combining (3.3), (3.4), and (3.5) we obtain (3.1).

Proof of Theorem 3. Let $f$ be holomorphic on a neighborhood of $\bar{B}$ and let $F=T_{\bar{\varphi}} f$. It is easily seen that

$$
D^{\alpha} F(z)=\int_{S} G_{n, \alpha}(\langle z, \xi\rangle) f(\xi) \overline{\varphi(\xi)} d \sigma(\xi),
$$

where $G_{n, \alpha}(\lambda)$ is a function defined in the unit disc by

$$
G_{n, \alpha}(\lambda)=\sum_{k=0}^{\infty}(k+1)^{\alpha} \frac{\Gamma(n+k)}{\Gamma(n) \Gamma(k+1)} \lambda^{k} .
$$

Thus, by Fubini's theorem

$$
\begin{aligned}
\int_{S}\left|D^{\alpha} F(\rho \xi)\right| d \sigma(\xi) & =\int_{S} D^{\alpha} F(\rho \xi) \psi(\rho \xi) d \sigma(\xi) \\
& =\int_{S}\left(\int_{S} G_{n, \alpha}(\langle\rho \xi, \eta\rangle) \psi(\rho \xi) d \sigma(\xi)\right) f(\eta) \overline{\varphi(\eta)} d \sigma(\eta) \\
& =\int_{S} f(\xi) \overline{\varphi(\xi) D^{\alpha} C\left[\bar{\psi}_{\rho}\right](\rho \xi)} d \sigma(\xi),
\end{aligned}
$$

where $\psi_{\rho}$ is a dilatation of $\psi$ (with the obvious definition).

From this it follows that

$$
\begin{aligned}
\left\|D^{\alpha} F\right\|_{1} & =\int_{S} f(\xi) \overline{\varphi(\xi) D^{\alpha} C\left[\bar{\psi}_{1}\right](\xi)} d \sigma(\xi) \\
& =\frac{2}{\Gamma(\alpha)} \int_{0}^{1}\left(\log \frac{1}{t^{2}}\right)^{\alpha}\left(\int_{S} D^{\alpha+1} f(t \xi) \overline{\varphi(t \xi) D^{\alpha} C\left[\bar{\psi}_{1}\right](t \xi)} d \sigma(\xi)\right) d t \\
& \left.\leq C\|\varphi\|_{\infty} \int_{0}^{1} \int_{S} \mid D^{\alpha+1} f(t \xi) \| D^{\alpha} \bar{\psi}_{1}\right](t \xi) \mid(1-t)^{\alpha} d t d \sigma(\xi) \\
& \leq C\|\varphi\|_{\infty}\left\|D^{\alpha} f\right\|_{1}
\end{aligned}
$$

by Lemma 3.1 with $\phi=\bar{\psi}_{1}$.

Finally, let $f \in H_{\alpha}^{1}$. Define $f_{k}(z)=f((1-1 / k) z), z \in B, k=2,3, \ldots$. Then by (3.6), we have

$$
\left\|D^{\alpha} T_{\bar{\varphi}} f_{k}\right\|_{1} \leq C\|\varphi\|_{\infty}\left\|D^{\alpha} f_{k}\right\|_{1} \leq C\|\varphi\|_{\infty}\left\|D^{\alpha} f\right\|_{1} .
$$

Passing to the limit, $k \rightarrow \infty$, we obtain $\left\|D^{\alpha} T_{\bar{\varphi}} f\right\|_{1} \leq C\|\varphi\|_{\infty}\left\|D^{\alpha} f\right\|_{1}$. This finishes the proof.

\section{REFERENCES}

1. A. Baernstein II, Analytic functions of bounded mean oscillation, Aspects of Contemporary Complex Analysis, Academic Press, New York, 1980, pp. 3-36.

2. F. Beatrous and J. Burbea, Characterization of spaces of holomorphic functions in the ball, Kodai Math. J. 8 (1985), 36-51.

3. __ Sobolev spaces of holomorphic functions in the ball, Pitman Research Notes, vol. 227, Pitman, London.

4. R. Coifman, R. Rochberg, and G. Weiss, Factorization theorems for Hardy spaces in several variables, Ann. Math. 103 (1976), 611-635. 
5. C. Fefferman and E. Stein, $H^{p}$ spaces of several variables, Acta Math. 129 (1972), 137-193.

6. D. Luecking, Forward and reverse Carleson inequalities for functions in Bergman spaces and their derivatives, Amer. J. Math. 107 (1985), 85-111.

7. L. Hörmander, L $L^{p}$-estimates for (pluri-)-subharmonic functions, Math. Scand. 20 (1976), 65-78.

8. M. Jevtić, Two Carleson measure theorems for Hardy spaces, Proc. of the Koniklijke Nederlandse Akademie van Wetenschappen Ser. A, vol. 92, 1989, pp. 315-321.

9. W. Rudin, Function theory in the unit ball of $C^{N}$, Springer-Verlag, New York, 1980.

10. F. Shamoyan, The Toeplitz operators on some spaces of analytic functions and a new characterization of the classes BMO, Izv. Akad. Nauk Armyan. SSR Ser. Mat. 22 (1987), 122-132.

MatematičKi fakultet, Studentski trg 16,11000 Beograd, Yugoslavia 